\title{
Efficiently Cooling Radiation-Damaged Silicon Pixel Sensors in the CMS HL-LHC Inner Tracker
}

Sam Bright-Thonney (skb93@cornell.edu), on behalf of the US-CMS TFPX Mechanics Group

The CMS Inner Tracker

- In the forward region of the inner tracker, pixel sensors are mounted on carbon fiber/foam support discs (Dees)

- Sensors will receive a lifetime dose of $2 \times 10^{16} \mathrm{n}_{\mathrm{eq}} / \mathrm{cm}^{2} / 1.2$ GRad TID

- Dark current increases as a function of fluence

$\rightarrow$ Positive feedback between temperature and current can cause thermal runaway in highly irradiated sensors

- Modules held near -30C using two-phase $\mathbf{C O}_{2}$ cooling to maintain performance and mitigate some effects of radiation damage

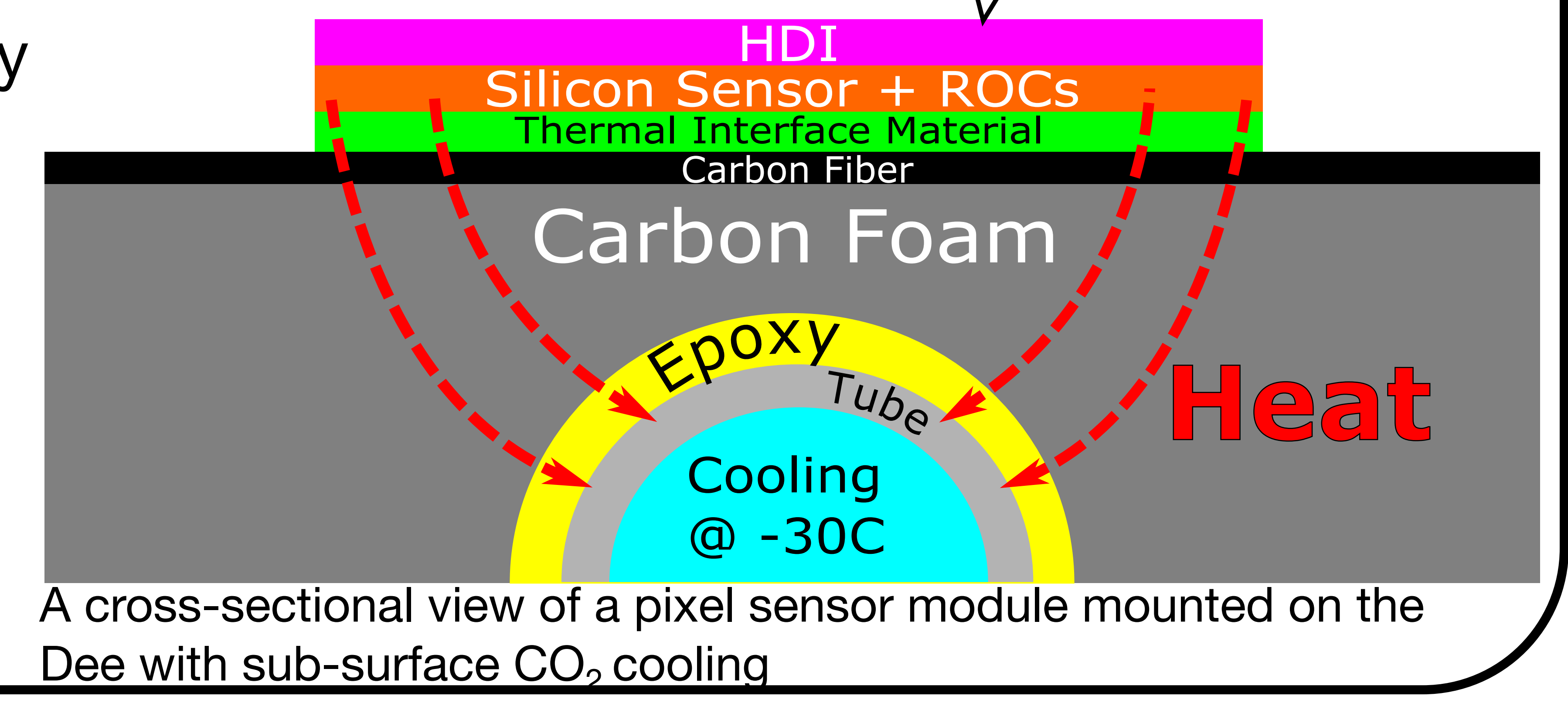

\section{Avoiding Thermal Runaway}

- A thin layer of thermal interface material (TIM) beneath each sensor helps move heat out of the module and toward cooling tubes

- TIMs must be:

Radiation hard - texture and thermal properties do not degrade

Conductive - at least $\sim 1 \mathrm{~W} / \mathrm{mK}$

Spreadable into thin, uniform layers

- TIMs chosen to ensure low net thermal resistance between sensor and cooling system

- Net thermal resistance determines operating temperature

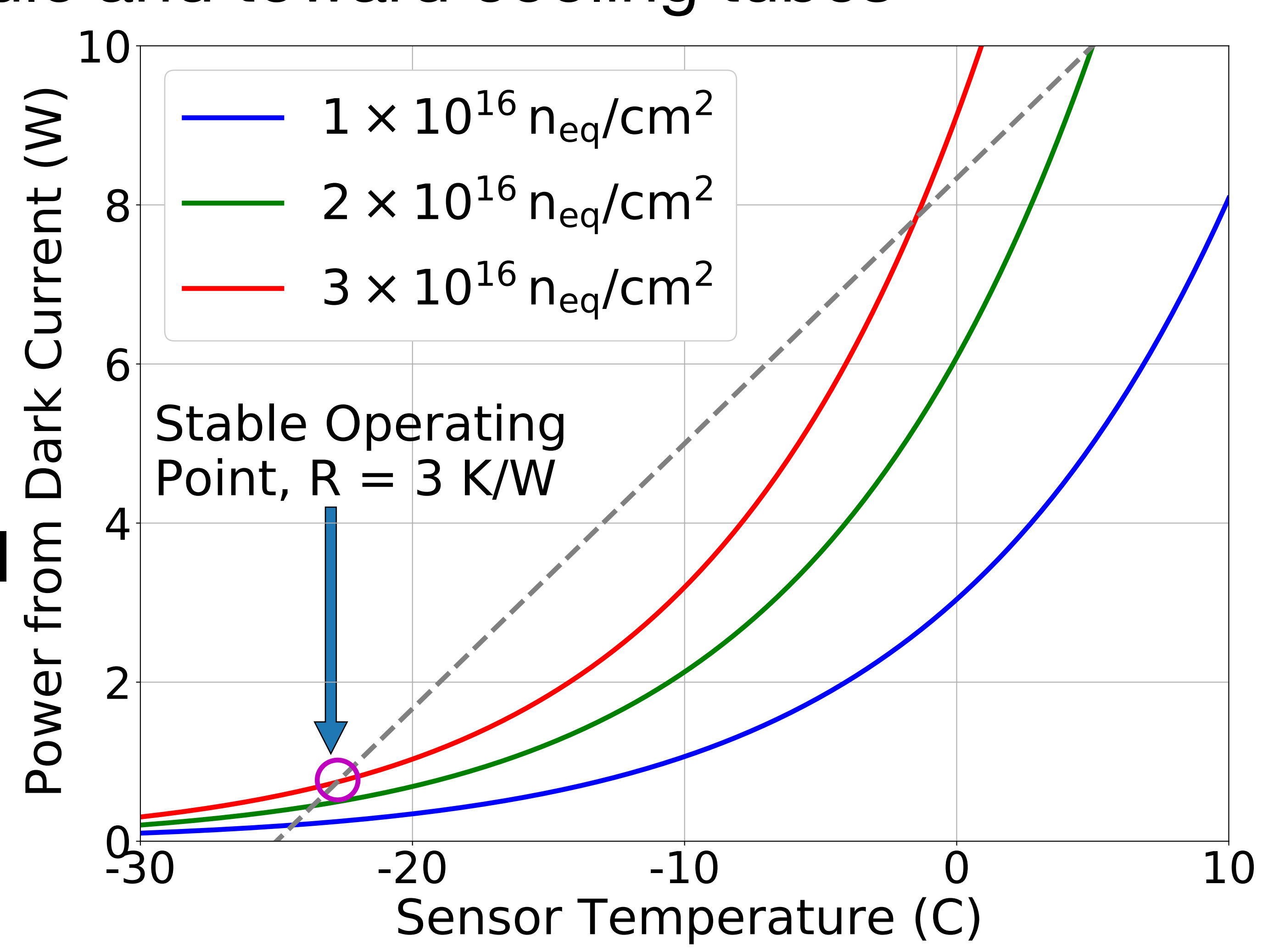

If $\mathrm{R}$ too large, no stable operating temperature $\rightarrow$ runaway!

$$
Q(T)=I\left(T_{\mathrm{ref}}\right) V_{\mathrm{bias}}\left(\frac{T}{T_{\mathrm{ref}}}\right)^{2} e^{-T_{A}\left(\frac{1}{T}-\frac{1}{T_{\mathrm{ref}}}\right)}
$$

\section{Radiation Hard Thermal Materials}

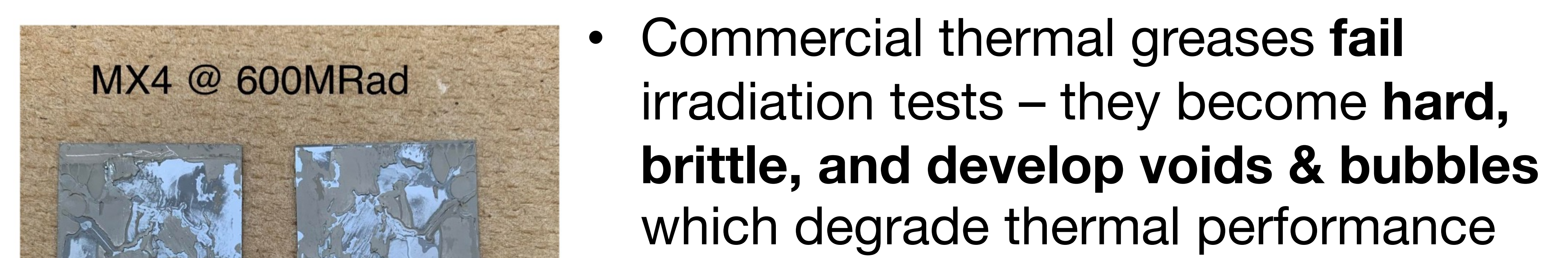
\&ubbles

- Polyphenyl Ether (PPE) -

high radiation vacuum grease, remains smooth \& spreadable up to 1.5 GRad
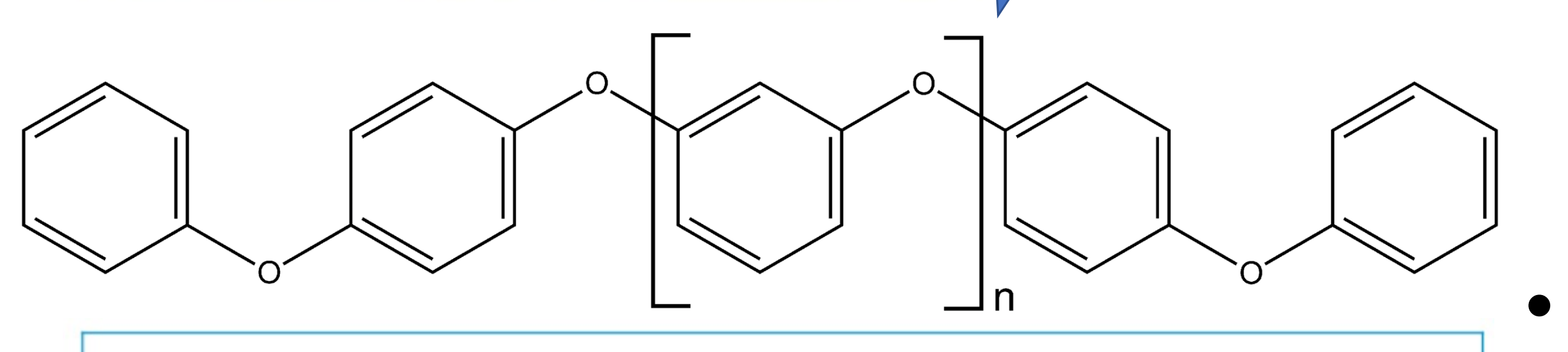

Material $k(W / m K)$

Pure Moresco PPE $\mathrm{PPE}+33 \%$ diamond $(20 \mathrm{um}) \quad \mathbf{0 . 3 3} \pm \mathbf{0 . 0 3}$ PPE $+70 \%$ diamond (20 um) $\quad 1.17 \pm 0.11$ PPE + Mixed-diameter diamond Measur

- TIMs that remain smooth enable module removal should it be necessary (repairs, damage, etc.)

- PPE is not thermally conductive $\rightarrow$ Increase conductivity by doping with micron-size diamond particles (current benchmarks are 30 and $70 \%$ diamond by mass, giving acceptable conductivities)

\section{Lab Testing PPE}

- In-lab simulation of thermal runaway using dummy "heater" modules Power is dynamically adjusted to mimic temperature-dependent dark current; cooling system similar to the Dee

- Future: runaway experiments using realistic dummy modules, cryogenic $\mathrm{CO}_{2}$ cooling, and a more sensitive thermal camera
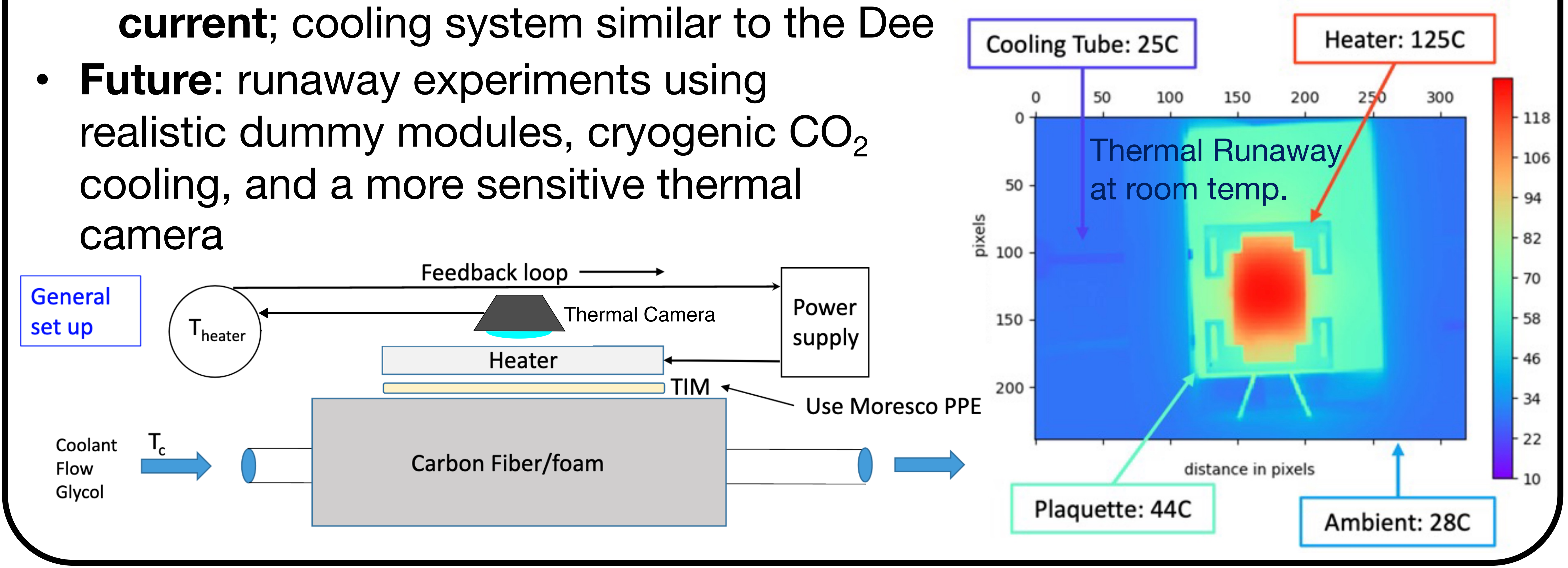